\title{
Experiences of Shanghai Medical Workers Who Assisted Wuhan in Fighting Against the COVID-19 Epidemic
}

\author{
Runv Zhou ${ }^{1 \dagger}$, Li Xu ${ }^{1 \dagger}$, Xueyan Li ${ }^{1 \dagger}$, Caiping Gao², Shikun Xu², Shengjie Liu ${ }^{1}$, Lin \\ $\mathrm{Qi}^{3}$, Tao Wang2* and Weiying Zhang1* \\ ${ }^{1}$ Shanghai East Hospital, Tongji University School of Medicine, China \\ ${ }^{2}$ Department of Emergency, Shanghai East Hospital, Tongji University School of Medicine/ \\ National Emergency Medical Team (Shanghai), Shanghai, China \\ ${ }^{3}$ College of Arts and Media, Tongji University
}

\section{Research Article}

Volume 4 Special Issue 1

Received Date: October 13, 2020

Published Date: October 29, 2020

DOI: $10.23880 /$ jidtm-16000S1-006

*Corresponding author: Weiying Zhang, MD, RN, Department of Nursing Department, Shanghai East Hospital, Tongji University School of Medicine, Shanghai 200120, China, Tel: +86 18217301505; Email: zwysh0701@163.com

Tao Wang, PhD, MD, Department of Emergency, Shanghai East Hospital, Tongji University School of Medicine/National Emergency Medical Team (Shanghai), Shanghai, China. Tel: +86 18930177382; Email: happywt0403@126.com.

†These authors contributed equally.

\section{Abstract}

Aim: This study aimed to explore the experiences of Shanghai medical workers who assisted Wuhan in fighting the COVID-19 epidemic and provide a reference for the development of later interventions for psychological stress.

Methods: We conducted a qualitative study using an interpretive description methodology. Fourteen Shanghai medical workers working in the ICU (intensive care unit) and Fangcang Shelter Hospital in Wuhan were selected for semi structured interviews in March 2020. Data collection and analysis were then performed.

Results: The fourteen interviewees experienced the following four theme categories and clusters to different degrees: Fighting without hesitation (anxious and nervous, happy and satisfied, calm); brief anxiety and fear (too nervous to sleep, worried about inadequate protection, shock and fear at first sight of the Fangcang shelter); adaptation and adjustment (not fighting alone, distracting oneself by doing what one likes to do, understanding and cooperation among team members, praise and support from patients); and feelings in the moment (continuing to work until the epidemic is under control, sense of professional achievement, avoidance).

Conclusions: Medical workers from other provinces and cities alleviated the shortage of medical treatment in Wuhan and ensured effective treatment for COVID-19 patients. Their psychological experiences and coping styles in different periods were quite different and were the true expressions of their emotions. This study will also provide them with further resources and help them gradually return to their original jobs.

Keywords: Experiences; Medical Workers; Mental Health; Phenomenological Study; SARS-CoV-2 


\section{Contribution of the Paper}

\section{What is already known about the topic?}

- In the wake of the COVID-19 pandemic, medical teams from various provinces and cities nationwide rushed to assist Wuhan during the Chinese Lunar New Year in 2020. This is the first time in China's history that such a large-scale response to a public health emergency occurred.

- Medical workers in COVID-19 regions had varying degrees of psychological problems, which has been reported in a number of studies.

- Little is known about the medical workers who assisted Wuhan in fighting against the COVID-19 epidemic, especially their real feelings during the epidemic.

\section{What this paper adds:}

- Different workplaces offer different experiences, depending on the work content, workplace, work partners and service objects.

- The semi structured interview not only gathered information about the work and life experiences and coping styles of Shanghai medical workers who assisted Wuhan in fighting against the COVID-19 epidemic but also gave them an opportunity to tell their stories.

- Families, hospitals and society have given great support to medical workers in Hubei Province.

- In the future, professional psychological assessment, psychological treatment and intervention should also be carried out to aid personnel in Hubei Province to help them return to normal as soon as possible.

\section{Background}

Infectious diseases have shaped the course of human history, resulting in more deaths than any other pathological cause [1]. In late December 2019, a cluster of patients with pneumonia of an unknown cause were reported to local healthcare authorities, and a novel coronavirus (SARSCoV-2) was identified as the etiology [2]. COVID-19 is a threat that can kill healthy adults and elderly people with existing health problems and is transmitted quite efficiently [3]. Current evidence indicates that SARS-CoV-2 may be spread by four major routes: direct or fomite contact, respiratory droplets generated during coughing or sneezing, face-oral, and airborne via aerosols [4].

By January 2020, the condition now known as COVID-19 was a major public health challenge in China. On January 23, China also raised its national public health response to the highest state of emergency (level 1 of 4 levels of severity in the Chinese Emergency System, defined as an "extremely serious incident") [5]. The Chinese government encouraged people to stay at home, discouraged mass gatherings, cancelled or postponed large public events, and closed schools, universities, government offices, libraries, museums, and factories [6].

From January 20 to February 8, there were 217 to 24,953 confirmed cases in Hubei Province, 55\% of which were located in Wuhan. The medical system in Wuhan was overburdened, personal protective equipment was seriously insufficient, patients with fever could not be treated in time, and cases of medical staff infection were frequently reported. Facing this large-scale infectious public health event, medical staff were under both physical and psychological pressure, which is an unprecedented challenge for healthcare workers $[7,8]$.

Since early January 2020, 31 deployed support medical teams, including 598 ICU doctors and 2,319 ICU nurses from other cities, have joined the ICU of designated hospitals in Wuhan to address the severe shortage of local medical resources. On January 24, the first 135 members of the emergency medical team rushed to Wuhan from Shanghai and quickly began their medical work in the ICU of Jinyintan Hospital. Jinyintan Hospital is a 3,300-bed grade-A tertiary hospital providing medical treatment during this outbreak. The Fangcang Shelter Hospital, also referred to as Fangcang Hospital, was also built in Wuhan. China has curbed the spread of COVID-19 and provided timely essential medical care to patients. It was believed that the rapid establishment and operation of Fangcang shelter hospitals was one of the key measures of controlling the epidemic in Wuhan [9]. The first three Fangcang hospitals were launched on February 5 and initially provided approximately 4,400 beds for mild-tomoderate COVID-19 patients.

As Bruce Aylward said, Chinese medical workers in the fight against the COVID-19 epidemic exude a sense of responsibility and collective action. They all have a mindset of fighting to complete the task [10]. A cross-sectional study of medical personnel in Wuhan showed that $22.4 \%$ experienced moderate interference, and $6.2 \%$ experienced severe interference after the initial outbreak. There is a need to better recognize that mental health needs are an important component of mobilizing a large-scale therapeutic response to sudden city-scale crisis scenarios [11]. Medical staff working in unfamiliar places faced many difficulties, such as a lack of knowledge of infection control and prevention, heavy workloads, and an associated risk of contamination [12]. Due to the uncertainty and uncontrollability of epidemic rescue work, coupled with the fact that the workers were far from home and their relatives, nurses in Hubei Province were under definite psychological pressure [13]. Stress and extreme fatigue could further challenge the immune system 
and increase susceptibility to COVID-19 [14].

\section{Methods}

\section{Study design}

A descriptive qualitative approach was applied. This approach is important and appropriate when a straightforward description of a phenomenon is required or information is necessary to develop interventions.

The research team consists of 8 members, including 2 psychological counselors, 3 senior hospital administrators and 2 qualitative research experts. There are 2 medical doctors and 2 individuals with master's degrees. Among them, 3 people rushed to Wuhan to assist with the frontline work. The researcher who conducted semistructured interviews with the subjects was the deputy leader of the National Emergency Medical Team (Shanghai), who comprehensively planned and managed the work and study of the personnel during their stay in Hubei Province.

\section{Establish interview outline and informed consent}

\section{Key questions:}

1) What was your motivation for and your psychological process when signing up for Wuhan?

2) What was your impression and feeling when you first arrived in Wuhan?

3) Recall the experience and feeling of finishing your first shift in Wuhan.

4) Was there any physical discomfort or change while working in Wuhan? If there was any physical discomfort or change, how did you cope with it and ease the discomfort caused by working in a high-risk and foreign area?

5) What are your deepest feelings now? What are your concerns and worries?

\section{Participants and Research Context}

The information of the participants is shown in Table 1.

\begin{tabular}{|c|c|c|c|c|c|c|c|}
\hline Number & Gender & Category & Professional direction & Age & $\begin{array}{c}\text { Time of arrival } \\
\text { in Wuhan }\end{array}$ & Wuhan workplace & $\begin{array}{c}\text { Wuhan } \\
\text { working days }\end{array}$ \\
\hline N1 & F & Nurse & Intensive care & 36 & February 4 & Fangcang hospital & 42 \\
\hline N2 & F & Nurse & Oncology care & 35 & February 4 & Fangcang hospital & 42 \\
\hline N3 & F & Nurse & Cardiac care & 29 & February 4 & Fangcang hospital & 42 \\
\hline N4 & M & Doctor & Pneumology & 38 & February 4 & Fangcang hospital & 42 \\
\hline N5 & F & Nurse & Intensive care & 28 & January 24 & ICU & 65 \\
\hline N6 & F & Nurse & Emergency care & 35 & January 24 & ICU & 65 \\
\hline N7 & M & Doctor & Infectious Diseases & 56 & January 24 & Infectious Clinic & 65 \\
\hline N8 & M & Nurse & Intensive care & 29 & January 24 & ICU & 65 \\
\hline N9 & F & Doctor & Cardiology & 41 & February 4 & Fangcang hospital & 42 \\
\hline N10 & M & Pharmacist & Pharmacology & 36 & February 4 & Fangcang hospital & 42 \\
\hline N11 & F & Nurse & Intensive care & 34 & January 26 & ICU & 56 \\
\hline N12 & F & Nurse & Intensive care & 23 & January 26 & ICU & 56 \\
\hline N13 & M & Nurse & Emergency care & 32 & January 26 & ICU & 56 \\
\hline N14 & F & Nurse & Intensive care & 30 & January 26 & ICU & 56 \\
\hline
\end{tabular}

Table 1: Participant characteristics.

\section{Data Collection}

After written consent, interviews were conducted with Shanghai medical workers who volunteered to participate in the study. They all assisted Wuhan in fighting against the COVID-19 epidemic. Medical workers from Shanghai to Hubei Province who worked in Jinyintan Hospital, Wuhan Third Hospital and Fangcang Hospital were selected as the research objects by objective sampling. The inclusion criteria were as follows: they could correctly express their recent working experience, voluntarily participate in this study, and sign the informed consent form. The interview location was the conference room of the hotel where the staff lived. The interview length was 40-60 minutes. With respect to precautions, the researcher and the interviewees wore surgical masks, and they were separated from each other by 1.5 meters.

\section{Data Analysis}

After the interview, the researcher listened to the recordings repeatedly and completed the transcription of 
the text within 24 hours. At the same time, the recorded email was sent to the second researcher in Shanghai, and the transcribed content was checked based on the recording. The two researchers reread the transcripts, extracted important statements, coded the transcripts separately, extracted important statements, and summarized the same and similar categories. Atlas.ti8 software was used to analyze highfrequency words. Themes were identified through a 6-step process: familiarization with the data, generating initial codes, searching for themes, reviewing themes, defining and naming themes, and producing the report [15]. To improve the accuracy and authenticity of the interview results, qualitative research experts need to review and guide the results.

\section{Ethical Consideration}

Ethical considerations are not involved. We obtained oral and written informed consent prior to all interviews. To protect the confidentiality of the participants, none of our records, including interview records, indicate their names or other identities. We present all the research results anonymously and only provided limited data access to the research team.

\section{Results}

In this part, we divide experiences and feelings into several different themes, according to different time periods (before departure, arriving in Wuhan, carrying out the first shift, current work, life, etc.), different types of medical staff (doctors, nurses, pharmacists) and different workplaces (ICU, Fangcang shelter). Each theme has its corresponding subtheme.

\section{Fight without Hesitation}

The rapid development of the epidemic situation poses a challenge to every medical staff member. Medical staff in Shanghai, especially those in relevant specialties, actively signed up for assistance in Wuhan. However, when faced with the unknown new coronavirus, everyone's psychological state differed during the period of waiting for departure.

Anxious and nervous: There was no effective treatment in the early stage. Who was needed now, who understood the respiratory system, and who knew about intensive care? That's exactly what I do. Whether we can go or not, I was also anxious at that time. Anyway, I really wanted to go, but what if I couldn't go? It was also a struggle (N4).

As a younger person, I was sure to move forward. That was no problem. I was more nervous and scared, because there was no special medicine for the treatment $(N 10, N 11)$.
Happy and satisfied: I'm very determined that I want to contribute my might. I am very happy because I can finally come (N3).

When I signed up, my husband was very angry that I did not consult with him. I am a nurse, and although my strength is limited, I hope to do such a thing, like a wish. I feel that I fulfilled a wish by coming here. I am quite satisfied (N5, N12).

Calm: I took the initiative to sign up at the first opportunity. This was a bound duty. Of course, my husband supported me very much $(N 1, N 12)$.

There was no tension and it was very calm. If we really go, we would be rich in materials and have strong logistical support, and there should be no danger. We felt stable (N1, N2).

I heard some news very early, and it was very contagious. It could be said that I was the only one on our team who knew about this disease (N4).

I was on vacation abroad and suddenly heard about the spread of the new coronavirus epidemic, and I decided that I must return home immediately (N9).

I received the notice to sign up for Wuhan, and I went back to Shanghai from Suzhou immediately. I am not really afraid. I just feel like I am going to work in another place (N14).

\section{Brief Anxiety and Fear}

At the time of departure, it was the most serious period of the COVID-19 epidemic in China, with strong infectivity and rapid transmission. At this moment, most of the medical staff have lingering fear, resulting in a series of physiological changes; there were many factors affecting the initial work experience.

Too nervous to sleep: My body is a bit unfit, and my sleep is a little disturbed. I watched the news, afraid of missing any information, and am in a nervous state (N3).

Tired? Somewhat, as there is stress and insomnia every day. I take sleeping pills (N12) and vitamins (N8) every day. After arriving at Wuhan Station, I felt that the air was starting to get dirty. At night, the room air conditioner suddenly turned on automatically, and I felt that the room was full of viruses (N9).

I often feel sleepy and feel sleepless (N10, N14).

My sleep quality is very terrible, about 2 hours a day, and even taking medicine is ineffective $(N 6, N 7, N 13)$.

Worry about inadequate protection: I did my best, but the protective clothing was too stuffy (N1).

I weas worried about whether the protective materials were enough and whether it was what I wanted (N2, N5). Fearing that there was no way to do my best, fearing that medicines were not available and there were no 
alternatives...I had been thinking about this (N10). Fear of becoming infected, being unsafe, and feeling a little anxious (N6,N11).

The nurses in Wuhan had been overworked for a long time, and they seemed to be very tired. I was worried that insufficient protection would lead to infection (N13).

Shock and fear at first sight of the Fangcang shelter: The beds were so densely packed that it was almost intensively fearinducing. Under such circumstances, I felt that the patients were going crazy. I was not worried about protection, but I was worried about not knowing what to do and feeling very bad and helpless. (N1).

I was worried about the security and police in Fangcang, and their awareness of prevention was not enough. Many patients in Fangcang were fathers, sons, and brothers, which was very unfortunate (N2).

Seeing the venue, I was really shocked, oh my gosh! There were many patients, which was scary, really scary. At that time, using my imagination was an impossible task, and my heart was still empty. Entering the cabin, I felt that I had entered another world (N4).

I was very scared because the patients were in very critical condition (N7, N12).

Fangcang could not be compared with a hospital; it was a warehouse. Before entering the warehouse, I was very nervous and frightened and thinking of death. After the first class came out, I felt that I came out alive, and I was no longer afraid (N9).

I'd work for 8 hours, afraid of falling down or dying (N13).

\section{Adaptation and Adjustment}

In Wuhan, medical staff was trying to adapt to achieve the best outcomes through self-regulation.

I was not fighting alone: I am a member of the rescue team. Many people are together. Behind me are hospitals and the government. The whole system is always guaranteed (N1).

I was very happy that the rescue team was together. I had a strong sense of security. If I had been the only one, I would feel very anxious (N3).

I learned that I was working with the pharmacist of the Jiangsu team. I felt very nervous and relaxed at once. I was not fighting alone, as we were a team (N10).

Distract yourself by doing what you like to do: We led the patients to a reading meeting in the Fangcang shelter. They moved me a lot (N3).

If there was something in the group, I would sign up immediately. I took all their jobs and did every job so that I could feel better (N4).

I felt lonely on my own. I had to sleep on my day off. Sometimes I watched TV or chatted with my family online
(N5).

There was always pressure. When I had a break, I watched chess games and comedy sketches and did math problems (N7).

Understanding and cooperation among team members: What I was most worried about now was the patients' emotional changes. First, the environment here was not very good. Second, the patients were all suffering from their treatment. Fortunately, our team could understand and tolerate each other, and everyone was more united here (N2). I felt that the nurses were working the hardest. These kids were born in the 1990s. Working in the intensive care unit was more risky. I was so touched by the fact that life and death were so different (N9).

My partner was cheerful, and we encouraged each other. He often told me jokes. I slowly adapted to the environment (N12).

Praise and support from patients: I was willing to talk to patients and listen to them. They were helpless. They were very grateful to us, saying that Shanghai's medical treatment was awesome, like seeing angels (N12).

I had adapted a lot now, and there was a little bit of enjoyment in the ICU because it was respected and appreciated by many patients (N13).

\section{Feelings in this Moment}

During this period, many medical staff members wanted to keep working until the end of the epidemic, which was very gratifying and moving. After witnessing the improvement of the epidemic situation in Wuhan, medical staff also found personal value from their work.

Keep working until the epidemic is under control: Take things as they come. At least we can't leave until the peak of the epidemic here is over and close to the end (N1).

There is no need for other people to rotate, because the pressure on the government here is already great (N4). The epidemic is not over. If we go back home, Jinyintan will definitely need someone to take over. Although I wish to go back home quickly, the next batch of people will need to explore again, and the team will work together. It doesn't seem worth it (N5).

I am getting better every day, doing my job well and controlling the epidemic well. I hope to go back early to witness the birth of my baby (N10).

Sense of professional achievement: I didn't expect to carry out the mission with the rescue team before. I gained rare experience. I broke through every step on my own (N3). After treating a serious patient, he gave his thumbs up to offer praise and gratitude. I felt very proud and had a sense of 
achievement (N6).

I feel that my career requires me to stand up in times of national crisis (N9).

Now, the mortality rate has dropped a lot, indicating that we have played a big role and have a great sense of value (N11,N12).

Avoidance: It's nothing special. Just like usual, it doesn't matter. I am ok (N8).

\section{Discussion}

The COVID-19 pandemic has alarming implications for individual and collective health and emotional and social functioning [16]. In describing their life experiences of fighting against COVID-19, healthcare providers pervasively emphasized a sense of responsibility to alleviate the suffering of patients and the need to work together to protect the entire country from the virus [17]. In addition, during the COVID-19 outbreak, 5.3\% of healthcare workers were screened for moderate to very severe depression, $8.7 \%$ for moderate to extremely severe anxiety, $2.2 \%$ for moderate to extremely severe stress, and $3.8 \%$ for moderate to severe psychological distress [18]. In a cross-sectional study among medical and nursing staff in Wuhan during the COVID-19 outbreak, $36 \%$ of the medical staff had subthreshold mental health disturbances, $34.4 \%$ had mild disturbances, $22.4 \%$ had moderate disturbances and $6.2 \%$ had severe disturbances [11].

The pneumonia clinic, department of infectious diseases and department of respiratory medicine in hospitals all over the country have been facing significant problems, such as long working hours, inadequate personal protective equipment and insufficient emotional support. Meanwhile, there was little awareness of novel coronavirus pneumonia. These issues had caused such adverse psychological outcomes, especially at the beginning of the outbreak. Unfortunately, frontline personnel sacrificed their own well-being and became infected or died, resulting in increasing psychological stress [19]. As with any natural disaster, epidemic, or other type of mass casualties, local healthcare capacity had been overwhelmed by the COVID-19 epidemic, which necessitated a request for external assistance at the national level [12]. The Chinese government was undoubtedly active and effective in controlling the spread of the epidemic. Medical teams from other provinces and cities as well as national emergency medical rescue teams have been dispatched to alleviate the shortage of medical care in Wuhan and ensure effective treatment for COVID-19 patients.

During the interview, participants sighed, twitched their mouth, cried, rubbed their hands together, or smiled. Their psychological experience and coping style in different periods of time were quite different, which was the true expression of their emotional reflection. They felt steady, firm from their calmness, and a little anxious when they set out. They felt scared when they arrived in Wuhan. They worked hard, supported each other, and tried to adapt to the new working environment.

Different workplaces offer different experiences, depending on the work content, workplace, work partners and service objects. The Fangcang Shelter Hospital is a novel model in response to the COVID-19 pandemic. It has provided timely medical care and isolation for COVID-19 patients [20]. COVID-19 patients with mild-to-moderate clinical characteristics were admitted to Fangcang Hospital. Quarantine is often an unpleasant experience for those who undergo it [21]. The medical workers of Fangcang Hospital provided emotional comfort and centralized treatment responses for patients. The working mode of medical workers is mainly teamwork, which reflects the spirit of cooperation. In this study, many subjects thought that the first entry experience was bad, but they felt safe because of the support of their peers. The experience also provided many entertainment outlets to regulate the mood of patients and enrich their isolated life, such as reading club, Baduanjin, and Xinjiang dance.

The first two groups of medical workers from Shanghai who worked in Wuhan ICUs seemed to have more difficulties in their personal experiences, such as obvious loneliness, an unwillingness to express themselves, and forbearance. N12 said, "On that day, I felt dizzy and nauseous when I was wearing protective clothing, and I kept breathing deeply, so I could not leave. If I handed over the patients I was in charge of two other people, it would increase their burden." In Jinyintan Hospital, the second northern ICU was taken over by the Shanghai medical team. From the perspective of data collection, medical advice processing, diagnosis and treatment, members were faced with unfamiliar environments and colleagues, which required a period of adjustment. The working habits were not uniform, and the handover was difficult. The protection resources were relatively insufficient, and it was inconvenient to drink water, eat and defecate. However, their sense of professional achievement was also higher than that of the Fangcang hospital medical staff. They felt the patients' gratitude for their care and experienced improved their self-worth in witnessing the miracle of rehabilitation.

Our research team believes that the 14 research subjects started the anti-epidemic work in Wuhan after they went to Wuhan and arrived in Wuhan. To date, the overall experience and response are positive and controllable, which is related to their own debugging ability and social support. The management department of the hospital provided material 
and spiritual help throughout the whole process to give them a sense of belonging. Wuhan also provided good hotel accommodations and popular catering as much as possible. People from all walks of life also frequently donated materials to ensure the convenience and comfort of medical staff working in Wuhan. The experience also made them feels valued and relieved their worries. Family support is an important category of emotional support. Parents, spouses, and children are the most important individuals in people's lives. Talking to family members at least once a day can effectively alleviate anxiety.

At the time of the writing of this article, all medical workers in Hubei Province have returned to Shanghai and been in medical quarantine and observation in five-star hotels. After a short period of adjustment, they gradually returned to their original jobs. We are facing unprecedented circumstances. This bio threat is the most serious global crisis of our generation. Medical workers have been on the frontlines of this fight, which has caused serious psychological damage. Will the negative experiences in Wuhan continue to have an impact? Do individuals' physiological and psychological states return to their initial levels? We therefore must support these individuals, and psychological counselors need to use more scientific methods of evaluation and intervention. The results of this study will also provide further resources and help. The research team will continue to follow up on the findings of this study.

\section{Strengths and limitations}

Due to the limitations of the epidemic, the interviewees in this study were involved with only three hospitals in Shanghai, and most of them came from the same hospital.

Among its strengths were the special interviewees. They provided in-depth information based on their various feelings and experiences.

\section{Conclusion}

This study aimed to explore the experiences of Shanghai medical workers who assisted Wuhan in fighting the COVID-19 epidemic and provide a reference for the development of later interventions for psychological stress. Due to the persistence and sense of responsibility of medical professionals, the medical workers left their permanent residences to go to unfamiliar places, regardless of their personal safety. The severe epidemic situation, heavy workload, and lack of adaptability in daily life, especially in the early stage of medical security material shortages, have greatly affected the psychological and physiological impact of the epidemic. Each participant reacted differently, worked actively, avoided deliberately, or cried in pain. Support from the hospital, society and families was the driving force for them to move forward bravely in the present moment. Further research is needed to determine whether this experience is valuable or has a negative impact on the future, or even if the physiological discomfort experienced persists for a period of time. We also suggest that hospital management departments develop personalized psychological interventions based on the results of this interview to help individuals return to work in Shanghai as soon as possible.

\section{Acknowledgments}

We sincerely thank the participating Shanghai medical workers who assisted Wuhan in fighting against the COVID-19 epidemic for their support and participation in this study.

\section{Funding Sources}

This work was supported by the following grants: Special subject of "Fighting Against the Epidemic Situation of COVID-19" (20TJBXKY15) (20TJBXKY16), Tongji University College of Arts and Communication, Shanghai East Hospital Institute of Disaster Medicine; and the Important Weak Subject Construction Project of Pudong Health and Family Planning Commission of Shanghai (PWZbr2017-13).

\section{References}

1. Huang C, Wang Y, Li X, Ren L, ZhaoJ, et al. (2020) Clinical features of patients infected with 2019 novel coronavirus in Wuhan, China. Lancet 395: 497-506.

2. Mccauley M, Minsky S, Viswanath K (2013) The H1N1 pandemic: media frames, stigmatization and coping. Bmc Public Health 13: 1116.

3. Gates B (2020) Responding to Covid-19 - A Once-ina-Century Pandemic? New England Journal of Medicine 382: 1677-1679.

4. Thomas JP, Srinivasan A, Wickramarachchi CS, Dhesi PK, Hung YM, et al. (2020) Evaluating the national PPE guidance for NHS healthcare workers during the COVID-19 pandemic. Clinical medicine (London) 20(3): 242-247.

5. Tian H, Liu Y, Li Y, Wu CH, Chen B, et al. (2020) An investigation of transmission control measures during the first 50 days of the COVID-19 epidemic in China. Science 368(6491): 638-642.

6. Chen S, Yang J, Yang W, Wang C, Bärnighausen T (2020) COVID-19 control in China during mass population movements at New Year. The Lancet 395: 764-766. 
7. Chen Q, Liang M, Li Y, Guo J, Fei D, et al. (2020) Mental health care for medical staff in China during the COVID-19 outbreak. The lancet Psychiatry 7: e15-e6.

8. Gao W, Sanna M, Tsai MK, Wen CP (2020) Geo-temporal distribution of 1,688 Chinese healthcare workers infected with COVID-19 in severe conditions-A secondary data analysis. PloS one 15(5): e0233255.

9. Chen S, Zhang Z, Yang J, Wang J, Zhai X, et al. (2020) Fangcang shelter hospitals: a novel concept for responding to public health emergencies. The Lancet 395(10232): 1305-1314.

10. Kupferschmidt K, Cohen J (2020) Can China's COVID-19 strategy work elsewhere? Science 367(6482): 10611062.

11. Kang L, Ma S, Chen M, Yang J, Wang Y, et al. (2020) Impact on mental health and perceptions of psychological care among medical and nursing staff in Wuhan during the 2019 novel coronavirus disease outbreak: A crosssectional study. Brain Behavior Immunity 87: 11-17.

12. Qiu H, Tong Z, Ma P, Hu M, Peng Z, et al. (2020) Intensive care during the coronavirus epidemic. Intensive care medicine 46(4): 576-578.

13. Mo Y, Deng L, Zhang L, Lang Q, Lio C, et al. (2020) Work stress among Chinese nurses to support Wuhan in fighting against COVID-19 epidemic. Journal of nursing management 28(5): 1002-1009.

14. Zhang Z, Liu S, Xiang M, Li S, Zhao D, et al. (2020) Protecting healthcare personnel from 2019-nCoV infection risks: lessons and suggestions. Frontiers of medicine 14(2): 229-231.

15. Devey Burry R, Stacey D, Backman C, Donia MB, Lalonde $M$ (2020) Exploring pairing of new graduate nurses with mentors: An interpretive descriptive study. Journal of clinical nursing 29(15-16): 2897-2906.

16. Pfefferbaum B, North CS (2020) Mental Health and the Covid-19 Pandemic. N Engl J Med 383: 510-512.

17. Liu Q, Luo D, Haase JE, Guo Q, Wang XQ, et al. (2020) The experiences of health-care providers during the COVID-19 crisis in China: a qualitative study. The Lancet Global health 8(6): e790-e798.

18. Chew NWS, Lee GKH, Tan BYQ, Jing M, Goh Y, et al. (2020) A multinational, multicentre study on the psychological outcomes and associated physical symptoms amongst healthcare workers during COVID-19 outbreak. Brain Behavior Immunity 88: 559-565.

19. Sun N, Wei L, Shi S, Jiao D, Song R, et al. (2020) A qualitative study on the psychological experience of caregivers of COVID-19 patients. American journal of infection control 48(6): 592-598.

20. Shang L, Xu J, Cao B (2020) Fangcang shelter hospitals in COVID-19 pandemic: the practice and its significance. Clinical Microbiology Infection 26(8): 976-978.

21. Brooks SK, Webster RK, Smith LE, Woodland L, Wessely $S$, et al. (2020) The psychological impact of quarantine and how to reduce it: rapid review of the evidence. Lancet (London) 395(10227): 912-920. 